\title{
PHOTOELECTROCHEMICAL PRODUCTION Of HYDROGEN
}

\author{
Eric Miller and Richard Rocheleau \\ Hawaii Natural Energy Institute \\ School of Ocean and Earth Science and Technology \\ University of Hawaii at Manoa \\ Honolulu, Hawaii 96822, USA
}

\begin{abstract}
In this year's Phase II photoelectrochemical hydrogen production research at the University of Hawaii, we have continued our emphasis on developing high-performance multi-junction hydrogen photoelectrodes based on low cost semiconductor and catalyst films. Significant progress was made in the advancement of amorphous silicon/germanium and copper indium gallium diselenide materials for solid-state photojunctions, and great strides were made in the research and development of the 'hybrid' photoelectrode concept, including important initial work on nanostructured wide-bandgap semiconductor films for the photoelectrochemical interface, particularly iron oxide. A new partnership was established with Duquesne University to take advantage of their demonstrated capabilities in fabrication of nanostructured films of photoactive $\mathrm{Fe}_{2} \mathrm{O}_{3}$ deposited by the spray-pyrolysis method; At the same time, an in-house investigation was initiated into the photoelectrochemical properties of reactively-sputtered iron oxide films. The Phase II work of this project is progressing on-schedule: To date, initial sets of prototype hybrid devices based on tandem amorphous silicon/germanium coated with corrosionresistant ITO and non-photoactive $\mathrm{Fe}_{2} \mathrm{O}_{3}$ films (sputtered at $\mathrm{UH}$ ) have been made, and prototypes using photoactive (deposited at Duquesne University) are currently under fabrication. Stable hydrogen photo-production was demonstrated in the hybrid prototypes fabricated with the sputtered iron oxide, but a voltage bias assist of $1 \mathrm{~V}$ was necessary. Near term plans include testing of the initial set of devices being fabricated with Duquesne's photo-active films for unassisted photo-production. We plan to follow analysis of the test results by a redesign of a second generation prototype with optimized interface and optical properties, for fabrication and testing by the end of the contract period.
\end{abstract}




\section{INTRODUCTION}

Under the sponsorship of the U.S. Department of Energy, in recent years the Thin Films Laboratory of the Hawaii Natural Energy Institute of the University of Hawaii has been developing high-efficiency, potentially low-cost, photoelectrochemical (PEC) systems to produce hydrogen directly from water using sunlight as the energy source. The main thrust of the work has been the development of integrated multijunction photoelectrodes, comprising low-cost semiconductor, catalytic, and protective thin-films deposited on low-cost substrates (such as stainless steel), for solar hydrogen production [Miller \& Rocheleau, 2001]. In the illustration of a generic hydrogen photoelectrode shown in Figure 1, sunlight shining on photoactive regions of the electrode produces electric current to drive the hydrogen and oxygen evolution reactions (HER, OER) at opposite surfaces.

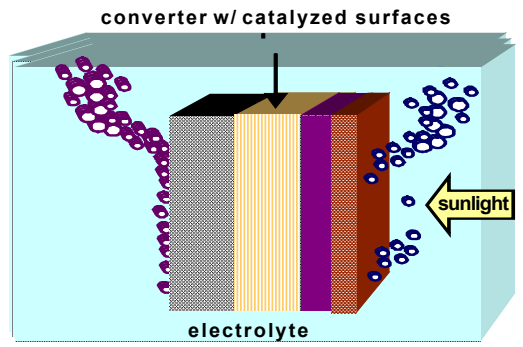

Figure 1. Integrated planar photoelectrode for hydrogen production.

In the conceptual design for a large-scale reactor shown in Figure 2a, arrays of photoelectrodes are arranged in tubular reactors, and electrolyte is circulated to extract the high-purity hydrogen and oxygen gases produced. As shown in Figure $2 \mathrm{~b}$, the integrated photoelectrodes would be installed within membranes along the tubes which allow ion exchange, but not gas flow to effect hydrogen and oxygen separation.

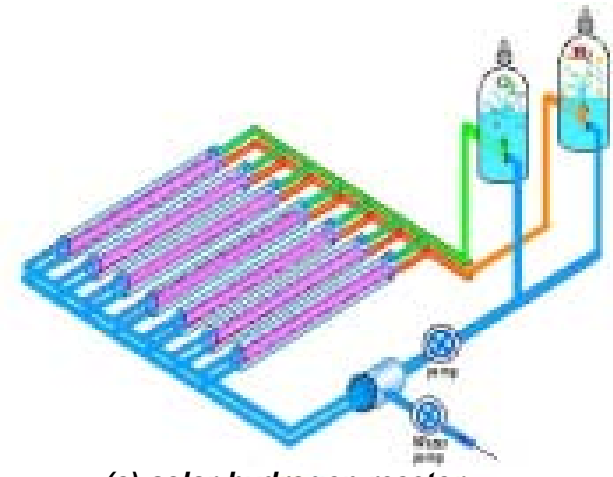

(a) solar-hydrogen reactor

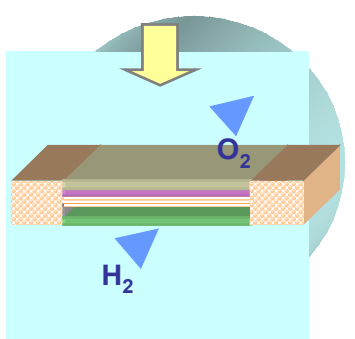

(b) tube cross-section

Figure 2. (a) Conceptual design of large-scale reactor with integrated photoelectrodes; (b) photoelectrode installation in collection tubes with membrane for gas separation.

In order to meet the DOE's goals, a PEC system must be low-cost, operate at solar-to-hydrogen (STH) conversion efficiencies greater than 10\%, and have long operating lifetimes in the corrosive aqueous electrolyte environment. In accordance with Equation 1 [Scaife 1980], the 
$10 \%$ STH efficiency corresponds to a hydrogen current density, $\mathrm{J}_{\mathrm{T}}$, of $8.1 \mathrm{~mA} / \mathrm{cm}^{2}$ at $\mathrm{AM} 1.5$ (based on the lower heating value for hydrogen).

$$
\underbrace{\eta_{\text {sтн }}(\%)}_{\begin{array}{c}
\text { solar-to-hydrogen } \\
\text { efficiency }
\end{array}}=\frac{1.23 \mathrm{~J}_{\mathrm{T}}}{\mathrm{E}_{\mathrm{T}}} \cdot 100=1.23 \underbrace{\mathrm{J}_{\mathrm{T}}}_{\mathrm{mA} / \mathrm{cm}^{2}} \text { for } \underbrace{\mathrm{E}_{\mathrm{T}}=100 \frac{\mathrm{mW}}{\mathrm{cm}^{2}}}_{\text {AM1.5 }}
$$

Numerous approaches involving a variety of semiconductors have been explored for hydrogen photoelectrolysis since the early 1980s, but none have successfully satisfied both the efficiency and stability criteria. The high voltage required to dissociate water (the $1.23 \mathrm{~V}$ dissociation potential, plus additional voltage drops associated with overpotential and system losses) and the corrosiveness of aqueous electrolytes have been major hurdles in this effort.

A photoelectrode design approach developed at UH incorporates multijunction thin-film photoconvertors (for high voltage) along with thin-film catalyst and protective layers (for stability). An important advance last year was the design of a "hybrid" photoelectrode structure combining solid-state junctions with stable, photoactive semiconductor films. This paper describes progress under Phase II of our current D.O.E. research contract, including work on amorphous silicon/germanium (a-Si:Ge), copper-indium-gallium-diselenide (CIGS) and hybrid designs incorporating iron oxide $\left(\mathrm{Fe}_{2} \mathrm{O}_{3}\right)$; It also proposes a new configuration combining the high efficiency of a CIGS design with the stability and simplicity of the hybrid concept.

\section{Photoelectrode Development}

Research at UH to design integrated photoelectrodes for directly splitting water into hydrogen and oxygen has evolved through several stages. This evolution is traced in Figure 3. As shown in Figure 3a, the original configuration used single-crystal, p-type silicon with an OER catalyst layer on the back surface, and scattered islets of platinum HER catalyst deposited onto the front (light-entering) surface. Here the semiconductor/ electrolyte junction formed the photovoltaic diode, and the HER islets facilitated current exchange. In the second stage photoelectrode, shown in Figure 3b, the front surface was evenly coated with Shottky-barrier metal, followed by HER catalyst layers thin enough for optical transmission, effectively replacing the semiconductor/liquid junction with a rectifying semiconductor/metal junction. The first two designs were fabricated using planar processing exclusively and were fully integrated on a single substrate. While these did result in a net energy savings, the voltage generated was below that required to split water, necessitating external biasing. Corrosion resistance was also poor.

The third-generation photoelectrode shown in Figure $3 \mathrm{c}$ incorporated several breakthrough features: First, the single-junction crystalline silicon diode was replaced by a triple-junction amorphous silicon diode (pinpinpin device deposited on a glass/CTO). Second, HER and OER thin-film catalysts of sputter-deposited NiMo and Fe:NiOx [Miller \& Rocheleau 1997], respectively, developed at UH specifically for low overpotentials and for long life in $\mathrm{KOH}$, were used. As described in previous reports. a small prototype based on this configuration achieved solar-tohydrogen efficiencies up to $7.8 \%$ [Rocheleau et al. 1998]. Since only catalyst surfaces were exposed to the electrolyte, excellent corrosion resistance was also achieved. The primary disadvantages were the use of separated electrodes and the need for external wiring. 


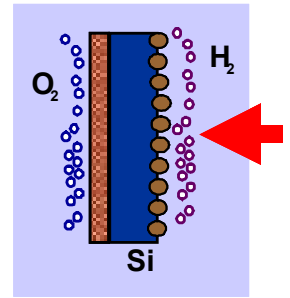

a) liquid junction

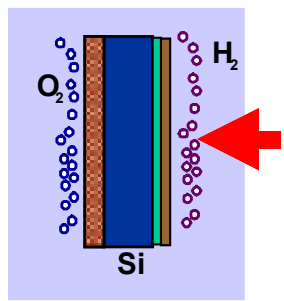

b) Schottky junction

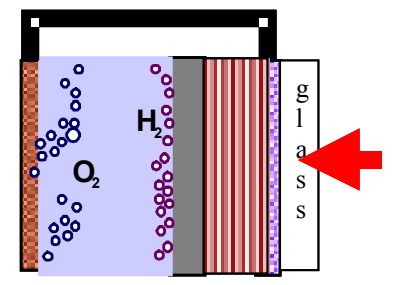

c) 3-junction a-Si:Ge on glass/ITO with separated electrodes

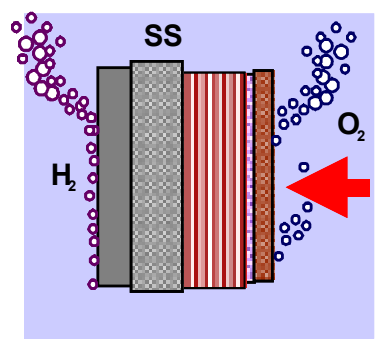

d) triple junction a-Si on SS

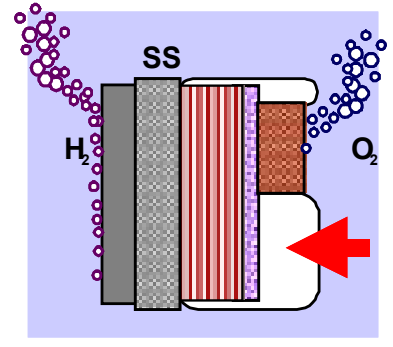

e) integrated planar photoelectrode design

Figure 3. Evolution at UH of planar photoelectrodes for solar-to-hydrogen conversion.

The external wiring was eliminated in the fourth-generation photoelectrode shown in Figure $3 \mathrm{~d}$. A stainless steel substrate was coated on the front side with a triple nipnipnip junction amorphous silicon/germanium (a-Si:Ge )diode and thin ITO and OER catalyst films, and on the back side with HER catalyst. The disadvantage was that light entered through the OER catalyst, leading to additional optical loss. With the metal oxide catalyst developed to date, films thin enough for good optical transmission offered little corrosion protection to underlying films, necessitating highly corrosion-resistant ITO and amorphous silicon. These photoelectrodes were fully integrated onto a single substrate and were fabricated using all planar processing techniques; however, the prototypes constructed all suffered from reduced efficiency due to the optical losses and from poor stability in $\mathrm{KOH}$ due to the thin protective/catalyst layer.

An important new feature in the fifth-generation photoelectrode structure pictured in Figure $3 e$ is the incorporation of a highly transparent and corrosion-resistant encapsulation film that does not need to be conductive. As in the previous configuration, the HER catalyst is sputter-deposited onto the back of a stainless steel substrate, and a-Si:Ge nipnipnip and ITO layers are deposited onto the front. Next, OER catalyst is sputter-deposited through a shadow mask onto a specified fraction of the diode's surface, and the encapsulation film is deposited through a second mask onto the remaining fraction to form a protected window for transmitting light. Light enters through the encapsulated window while current is transported through the catalyst surface; this precludes the need for ultra-thin/transparent OER catalyst films. High efficiency requires modest concentration to focus the light onto the transparent region of the surface. This design is fully integrated onto a single substrate using a sequence of straightforward planar processing steps, and a significant advantage is that only high-stability surfaces are exposed directly to electrolyte. Disadvantages include the added component of lateral current collection, added fabrication complexity at the front surface, and front-surface seams (e.g., catalyst and encapsulated window edges) that could result in device degradation over long exposure to electrolyte. 
Another major advantage in the integrated photoelectrode with encapsulation is the opportunity for stacking the series-connected multijunction solid-state cells side-by-side as well as monolithically. As a result, higher-efficiency materials such as copper-indium-gallium-diselenide (CIGS), which cannot stack monolithically, can be used as an alternative to a-Si:Ge. Figures 4a and $4 \mathrm{~b}$ show, respectively, the basic design structures for multijunction encapsulated photoelectrodes with monolithically stacked a-Si:Ge and with side-by-side stacked CIGS. Both Figure 4 device structures, in particular the CIGS device in Figure 4b, represent a significant increase in fabrication complexity over previous generation photoelectrodes. In both, lateral collection of current through the ITO represents an efficiency-reducing loss. The reduced surface area of the front surface catalyst also degrades hydrogen conversion performance. Despite the disadvantages, enhanced stability through encapsulation of underlying semiconductor layers is an attractive feature. Also of import, both the a-Si:Ge and CIGS photoelectrode systems have the potential for low cost based on the very thin semiconductor layers involved and on compatibility with high-throughput manufacturing processes.
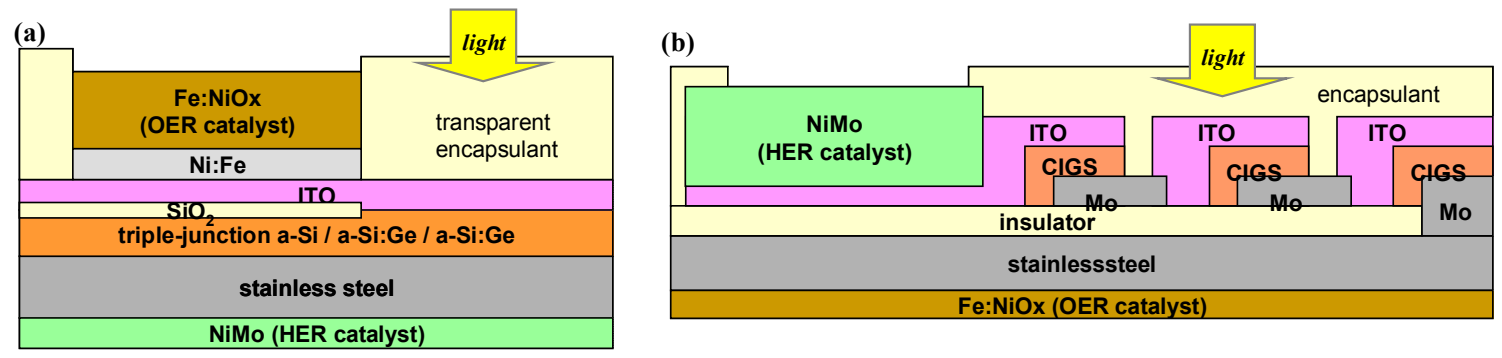

Figure 4. Structure of encapsulated triple-junction photoelectrode using (a) a-Si:Ge ; (b) CIGS.

As discussed in the device modeling section below, even with the additional current-collection and catalyst area-reduction losses, model results indicate solar-to-hydrogen efficiencies in excess of $10 \%$ for the CIGS photoelectrode; unfortunately, predicted efficiencies drop below $10 \%$ for the encapsulated a-Si:Ge structure. This is explained in part by the higher photovoltaic efficiency in CIGS, which can approach 19\% [Tuttle et al., 1995], compared with a-Si:Ge, which has demonstrated peak efficiencies up to only 13\% [Wang et al. 2001]. Another factor is the comparative losses associated with the reduced front surface catalyst areas in the two configurations. For optimal PV efficiency in a-Si:Ge cells, light should enter through the p-layer, dictating OER catalyst on the front surface. In CIGS cells, where light enters through the $n$ layer, the front surface must be catalyzed for the HER. Since HER overpotentials are significantly lower than those for the OER with available catalysts, reduced HER front-surface catalyst in the CIGS cells represents a less severe loss.

\section{Photoelectrode Modeling}

As detailed in previous reports, analyzing the operation of hydrogen photoelectrodes requires a complex integration of photovoltaic, optical, and electrochemical phenomena. An important part of the UH research has been the development of integrated models combining these effects [Rocheleau \& Miller, 1997]. The elements of these models, based on a lumped-circuit model of a photocell in series with a current-dependent electrochemical load, are shown in Figure 5a for a triple-junction photocell. In this analysis, the dark diode characteristics are estimated from the performance of high-quality devices reported in the literature. The light JV characteristics of the photocells are calculated assuming superposition with light-generated currents and complete 
absorption of photons with energies above the optical gap and complete transmission of those below it. The electrochemical model (shown on the right-hand side of Figure 5a) uses the Butler-Volmer relation to describe the current-dependent overpotentials due to charge transfer kinetics at the electrode surfaces, and included additional resistances for the potential drop due to ion transport through the electrolyte. Kinetic parameters used in the model are based on values achieved in thin-film, sputter-deposited catalysts developed at $\mathrm{UH}$. The decomposition potential $\left(V_{e q}\right)$, along with system resistances $\left(R_{e c}\right)$, are taken from the literature.
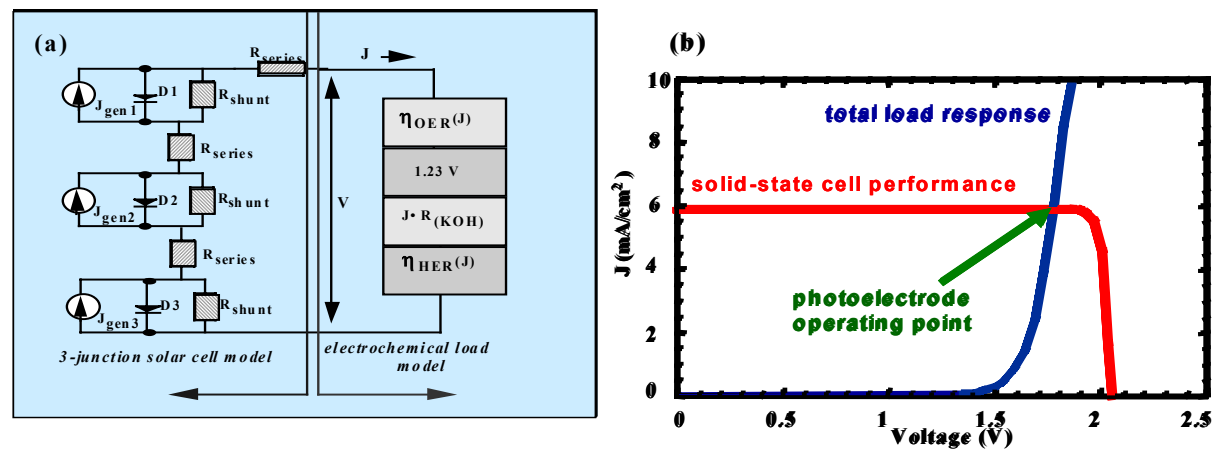

Figure 5. (a) Model of triple-junction photoelectrode; (b) load-line analysis to determine photoelectrochemical operating point.

Outputs from this integrated analysis include the current-voltage (JV) curve of the photocell and the load curve of the electrochemical cell. The intersection of these two curves defines the operating point of the system, as illustrated in the load-line analysis plot in Figure $5 \mathrm{~b}$. For each semiconductor system-amorphous silicon, Group III-V crystalline cells, and CIGS-different combinations of bandgaps and electrochemical parameters were examined to identify designs with the highest expected hydrogen production rates. Even with highly optimistic assumptions for the diode characteristics (i.e., small reverse saturation currents), the analysis showed that semiconductor bandgaps greater than $1.9 \mathrm{eV}$ are required to generate voltages sufficient for direct water-splitting when single-junction photoelectrodes are considered. The analysis also showed that any loss in junction or catalyst performance in such systems would result in large performance losses, requiring even higher bandgaps to provide a reasonable probability of stable operation. The poor absorption of the solar spectrum with such high bandgaps results in low solar-to-hydrogen conversion efficiencies.

Of the materials evaluated in multijunction photoelectrodes, those based on crystalline Group III-V heterojunctions showed the highest theoretical efficiencies, with values as high as $20 \%$ in tandem structures. The high cost of these materials, however, makes them impractical for commercial PEC systems. Because of their good voltage match, solar-to hydrogen conversion efficiencies for the much lower-cost multijunction a-Si:Ge structures were predicted to be as high as $10 \%$. Moreover, it was predicted that conversion efficiencies approaching $15 \%$ are possible using the encapsulated photoelectrode designs with multijunction copper-indiumgallium-diselenide (CIGS) cells stacked in a side-by-side configuration [Miller\&Rocheleau 2001]. Of significance, both the a-Si:Ge and CIGS-based photoelectrodes can be fabricated on lowcost substrates such as metal foils. They have further potential for low cost based on the very thin semiconductor layers involved and on compatibility with high-throughput manufacturing processes. 


\section{Hybrid Photoelectrode Concept}

As an alternative approach to both the integrated a-Si:Ge and CIGS photoelectrode structures, which utilize photo-generation in multiple connected solid-state junctions, UH last year developed the conceptual design for a hybrid solid-state/PEC photoelectrode incorporating current-matched solid-state and electrochemical photojunctions. This design was in part motivated by recent advances in semiconductor materials, such as dye-sensitized $\mathrm{TiO}_{2}, \mathrm{WO}_{3}$, and $\mathrm{Fe}_{2} \mathrm{O}_{3}$, for photoelectrochemical applications. The design process was greatly facilitated by integrated photovoltaic/photoelectrochemical modeling efforts. A fundamental design strategy for producing high efficiency in solid-state multijunction amorphous silicon/germanium devices involves careful current matching in each of the junctions by adjustment of the absorption spectra through bandgap and i-layer thickness tailoring. The basic device structure for highefficiency $(12.7 \%$ ) triple junction a-Si:Ge solar cells developed at the University of Toledo [Wang et al. 2001], shown in Figure 6a, incorporates three component nip cells, with i-layer bandgaps ranging from 1.79 to 1.55 to $1.39 \mathrm{eV}$ in the top, middle, and bottom cells, respectively. The top layer conductive-transparent oxide (CTO) is used to collect front-surface current, while the back surface reflector layer is used to reflect back photons and boost quantum efficiency (QE). Figure 6b shows the QE curves for this device, along with the AM 1.5 integrated currents for the component cells, and for the total combined cells. Since device current will be limited by the lowest-current cell in the series-connected stack, current-matching in the component cells is critical for good performance. In current-matching, the total device integrated current should be equally divided into each of the component cells to fully utilize the total photogenerated carriers.

(a)

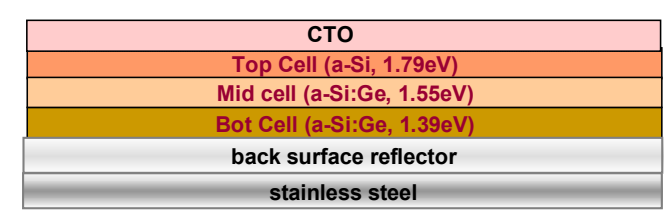

(b)

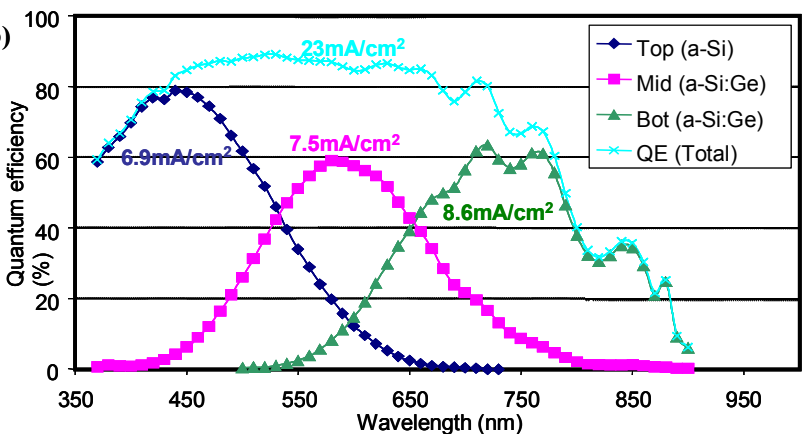

Figure 6. (a) Structure of the Toledo $12.7 \%$ triple-junction a-Si/a-Si:Ge/a-Si:Ge solar cell; (b) AM 1.5 quantum efficiencies and integrated currents for component cells and multijunction device.

As seen Figure $6 \mathrm{~b}$, the top nip junction in the $12.7 \%$ device is designed to absorb most strongly in the $350-500 \mathrm{~nm}$ range. In principle, this top cell could be replaced or amended by a PEC junction with strong absorption in a similar range to form a water-splitting photoelectrode for hydrogen production. As shown in Figure 7, this hybrid photoelectrode can be fabricated on stainless steel with the back surface catalyzed for the HER, and the front surface deposited with a solid-state multijunction followed by a CTO interface layer, then by a photoactivesemiconductor layer. The semiconductor top layer, which forms a PEC junction in electrolyte, must have appropriate conduction band alignment for the OER, must be strongly absorbing in the $350-500 \mathrm{~nm}$ region for current-matching to the underlying solid-state junctions, and must be stable. Significant advantages of the hybrid design over the encapsulated design from Figure $3 e$ include elimination of the lateral current collection, reduction in OER overpotential, enhanced stability due to the thick uniform outer layer, and greatly simplified geometry for fabrication ease. 
photoelectrochemical junction with electrolyte

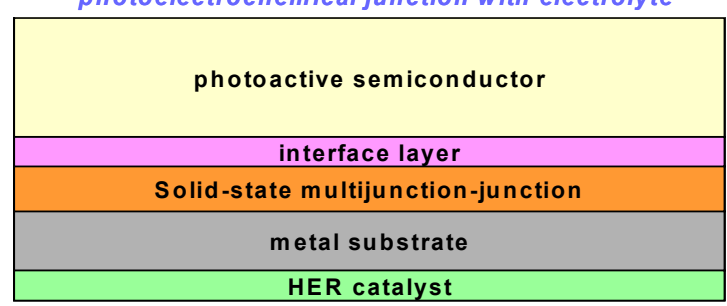

Figure 7. Basic structure for a hybrid solid-state/photoelectrochemical photoelectrode.

Research advancements in dye-sensitized titanium dioxide $\left(\mathrm{TiO}_{2}\right)$, tungsten trioxide $\left(\mathrm{WO}_{3}\right)$, and iron oxide $\left(\mathrm{Fe}_{2} \mathrm{O}_{3}\right)$ indicate that these may be suitable candidate materials for the hybrid photoelectrode. Figure 8, for example, shows the quantum efficiencies (stated in I.P.C.E, or incident photon conversion efficiency) of $\mathrm{TiO}_{2} \mathrm{PEC}$ junctions as a function of sensitizing dye [Graetzel 1999]. The junction sensitized with the $\mathrm{RuL}_{3}$ dye exhibits peak absorption near $500 \mathrm{~nm}$, consistent with the requirements for the hybrid photoelectrode. Interestingly, photoelectrochemical photovoltaic cells based on dye-sensitized $\mathrm{TiO}_{2}$ have been successfully demonstrated using the $\mathrm{I}^{-} / \mathrm{I}_{3}^{-}$couple [SFIT 1996]. Unfortunately, however, inherent instability in all currently known dyes under OER conditions make this versatile material unsuitable for the hybrid hydrogen photoelectrode, at least at this time. Continued research into new dyes that remain stable under oxidation is reportedly underway [Koningstein 1995].

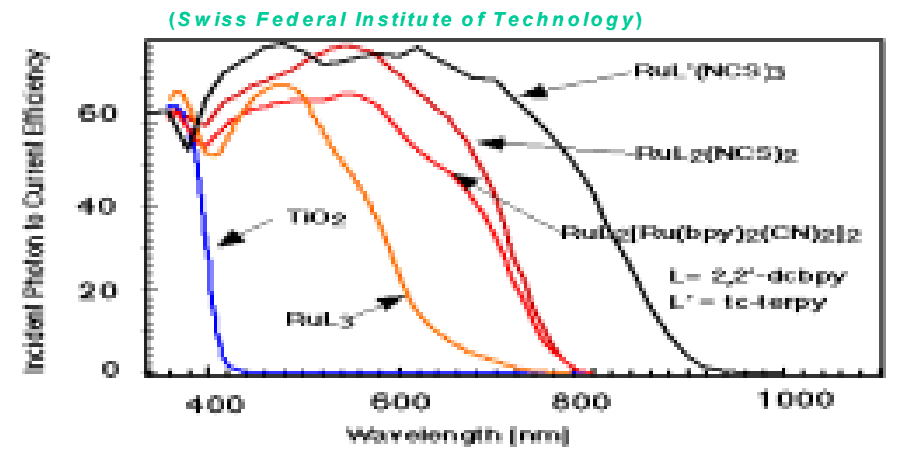

Figure 8. Quantum efficiencies in dye-sensitized $\mathrm{TiO}_{2}$ photoelectrochemical junctions.

Hybrid photoelectrode development has become an important focus of our PEC research at UH. During this research, we have continued to develop integrated electrochemical/optical models to identify materials and multijunction electrode designs with potential for high efficiency and stability (e.g., the encapsulated series-connected CIGS triple junction photoelectrode, and the more recent hybrid designs) and in conjunction, we have fabricated and tested various photoelectrode prototypes engineered to maximize efficiency and stability for given material properties. Specific goals, milestones, and achievements for this year's Phase II effort are described in the following sections.

\section{GOALS AND MILESTONES}

The long-term goal of PEC research at UH is the development of high efficiency, low-cost photoelectrochemical processes for the direct production of hydrogen. Toward this goal, 
integrated electrochemical/optical models have been used to identify materials and electrode designs with potential for high efficiency, and prototype photoelectrodes, engineered to maximize efficiency and stability for given material properties, have been fabricated and evaluated. The major emphases of the long-term objective can be summarized as follows:

- Development of a high efficiency, cost-effective photoelectrochemical process for the production of hydrogen (focusing on low-cost materials, such as SS foil, a-Si:Ge, CIGS, etc.)

- Engineering of multijunction photoelectrodes, based on low-cost materials, with stable aqueous interfaces that match energy requirements for hydrogen production.

- Producing optimized photoelectrodes suitable for eventual commercial-scale use.

Specific work for this funding period (Phase II of the current contract) falls under three main tasks. These tasks, along with projected milestones (based on a one-year project period with "Quarter 1" starting on 1 September 2001) are summarized as follows:

- TASK 1: Multijunction a- Si:Ge Photoelectrodes:

- Qtr 1: Complete evaluation of triple junction amorphous silicon photoelectrodes.

- TASK 2: CIGS Photoelectrode Development:

- Qtr 2: Establish industry partners.

- Qtr 2: Adapt integrated model to CIGS photoelectrodes.

- Qtr 3: Upgrade in-house CIGS fabrication facility.

- Qtr 4: Fabricate and test CIGS photoelectrodes.

- TASK 3: Hybrid Photoelectrode Development:

- Qtr 1: Establish partnerships to accelerate development of $\mathrm{TiO}_{2}, \mathrm{WO}_{3} \& \mathrm{Fe}_{2} \mathrm{O}_{3}$.

- Qtr 2: Adapt integrated models to hybrid systems.

- Qtr 3: Establish and design hybrid system for initial testing.

- Qtr 4: Fabricate and test hybrid photoelectrode.

In terms of Task 1 above, we decided, as a result of our Phase I work last year, to focus more on the development of higher-efficiency CIGS devices, and to terminate efforts to develop the triple-junction a-Si:Ge counterparts. As previously reported, the integrated triple-junction aSi:Ge photoelectrode, using state-of-the-art a-Si:Ge processing to obtain PV efficiencies around $13 \%$, would be expected to split water with solar-to-hydrogen efficiencies between $6 \%$ and $8 \%$, based on integrated models including all losses. On the other hand, CIGS diodes using state-ofthe-art processing can have PV efficiencies exceeding 18\% [Tuttle et al. 1995], and according to our models, solar-to-hydrogen efficiencies exceeding $10 \%$ are readily achievable using the CIGS devices. There are added complexities in the fabrication of the side-by-side CIGS photoelectrodes, as seen in Figure 4b vs. 4a; but we feel that the disadvantages in complexity are outweighed by the potential for higher efficiency in the integrated CIGS photoelectrodes. As a result, we've initiated accelerated efforts to identify and develop model photoelectrode systems based on high-efficiency CIGS.

Also as a result of our Phase I work, we've place a strong emphasis on developing model photoelectrode systems based on novel hybrid configurations incorporating wide bandgap semiconductor films (low-cost, highly-stable) deposited on top of optically-tailored a-Si:Ge tandem devices, as described in the Introduction section. A more detailed description of the individual tasks, along with progress in achieving the delineated milestones is presented in the Progress Status section below. As of the D.O.E. Hydrogen Review meeting in May 2002, all 
milestones through quarter 3 (ending 31 May 2002) have been met, putting the project onschedule.

\section{PROGRESS STATUS}

Consistent with the tasks delineated in the previous section, major activities this funding period (Phase II of the current contract) have focused on: (1) completing our comparative evaluation of triple-junction a-Si:Ge vs. CIGS photoelectrode configurations; (2) accelerating efforts toward high-efficiency CIGS devices; and most importantly, (3) the design and development of the hybrid photoelectrode configuration using low cost semiconductor films (such as $\mathrm{Fe}_{2} \mathrm{O}_{3}, \mathrm{WO}_{3}$ or $\mathrm{TiO}_{2}$ ) in conjunction with underlying a-Si:Ge devices. Progress in our a-Si:Ge research (relevant to both Task 1 and Task 3), CIGS research (Task 2) and hybrid development (Task 3) is described below:

\section{Amorphous Silicon/Germanium Research}

Continued research to develop amorphous silicon/materials and devices during Phase II of our current PEC program has focused on explaining results from Phase I experiments with integrated triple-junction photoelectrodes, and on developing material systems for hybrid photoelectrode designs. The major milestones achieved to date in our Phase II a-Si:Ge work can be summarized as follows:

- Completed experiments to determine device stability and yield as a function of metal-foil substrate material and surface. Established SS foil type with optimal yields.

- Fabricated initial set of double- and triple-junction a-Si:Ge devices for use in hybrid photoelectrode tests (University of Toledo sub-contract).

- Deposited interface layer of corrosion-resistant ITO onto the a-Si:Ge samples.

A key milestone last year was the fabrication and testing of integrated amorphous silicon/germanium photoelectrodes configured as shown in Figure $4 \mathrm{e}$. The photoelectrode structure, fabricated onto 1" x 1" stainless steel foil, incorporated the University of Toledo's highefficiency $(12.7 \%)$ triple junction cells, NiMo HER thin-film catalyst, and EP39-2 polymer encapsulant. Prototype devices were tested in $1 \mathrm{~N} \mathrm{KOH}$ under simulated solar illumination (ELH lamps) and outdoors under sunlight. In all devices, gas bubbles were observed evolving from the catalyst surfaces, as seen in Figure 9. Unfortunately, as reported last year, significant shortcomings in both efficiency and stability were observed over the course of these tests.

(a)

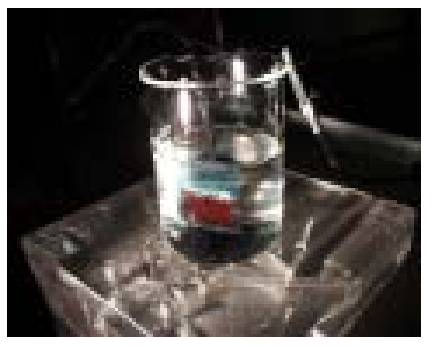

(b)

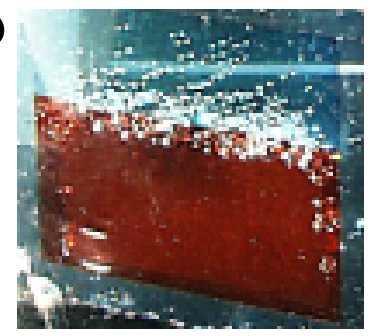

Figure 9. (a) apparatus for testing encapsulated triple-junction a-Si:Ge photoelectrodes; (b) gas evolution observed at photoelectrode surface. 
The triple-junction a-Si:Ge cells fabricated at the University of Toledo exhibited as-deposited photovoltaic efficiencies up to $12.7 \%$ and open-circuit voltages up to $2.3 \mathrm{~V}$. Based on these parameters, our models indicated solar-to-hydrogen efficiencies ranging from $6 \%$ to $8 \%$ were expected. The loss from the electrical efficiency and range is based on lost area and estimated ranges for optical, electrical, and electrochemical losses. However, only $25 \%$ of the devices tested under outdoor conditions demonstrated hydrogen efficiencies exceeding $1 \%$, and the highest observed efficiency was $2.5 \%$. This disappointing result was traced to degradation in the solar cell performance as a result of the processing steps used in photoelectrode fabrication.

This year, in order to better understand the yield and stability problems encountered in our solidstate multi-junction a-Si:Ge devices, we initiated, in conjunction with the University of Toledo, a series of experiments to evaluate the effects of the underlying metal substrate. Triple junction devices were fabricated onto stainless-steel and nickel foil substrates with different compositions and surfaces finishes. A "standard" recipe (University of Toledo) was used to deposit triple-junction layers onto 1" $x$ 1" substrates, then onto each substrate, 10 ITO (indiumtin oxide) dots were deposited to form the contacts for 10 discrete devices. Current-voltage characterizations (JV) under 1-sun conditions were performed on each of the discrete devices, and yield for each substrate was determined as the percentage of operational devices (based on fill factor thresholds) with respect to total devices. Table 1 below summarizes the photovoltaic performance and yield results from these experiments. Included are results from nickel (Ni) foil, stainless-steel 302 (SS 302) foil (used in the Phase I triple-junction photoelectrode devices), stainless-steel 304 (SS 304), and a proprietary-composition stainless steel (SS UT) used by the University of Toledo in their photovoltaic research.

It is clear from results in Table 1 that device yield depends strongly on the metal substrate. A particularly significant result is that the SS-302 foil used in the Phase I triple-junction electrodes consistently exhibited photovoltaic yields less than $80 \%$. This explains, at least in part, the yield problems observed in the photoelectrodes fabricated onto these substrates. Another significant result seen in Table 1 is the 100\% photovoltaic yield in devices formed on the SS-UT substrates. On the basis of this result, the SS-UT material was selected as the substrate of choice for the remainder of all Phase II work in the development of the hybrid structures.

To date, one series of tandem a-Si:Ge devices has been fabricated by the University of Toledo (under sub-contract) onto SS-UT foil for use in initial hybrid photoelectrode experiments, as further described in the Hybrid Photoelectrode Development section. Corrosion-resistant ITO [Chin 2000] was deposited onto the a-Si:Ge devices at UH In preparation for a photoactive layer application. The measured quantum efficiencies for layers in a typical tandem cell (based on the bottom two layers of a triple junction device) are shown in Figure 10.

\section{CIGS Development}

Key milestones last year during Phase I of this work included demonstration of our CIS (copper indium diselenide) device fabrication capabilities, and completion of our preliminary investigation of CIS photoelectrodes, including corrosion testing of CIS materials, and fabrication of a mechanically interconnected test structure simulating the side-by-side series connected configuration (Figure 4b). This year, during Phase II, we concentrated on establishing key industrial partnerships and acquiring the essential expertise for upgrading our facilities to fabricate the higher-efficiency CIGS (copper indium gallium diselenide) for use in advanced photoelectrode designs. Important milestones achieved to date in the CIGS research effort have included: 
- $\quad$ Maintained present and established new university and industrial partnerships.

- $\quad$ Adapted models to photoelectrodes using series-connected CIGS cells.

- $\quad$ Tested series-connected CIGS based photodiodes \& used results for model validation.

- $\quad$ Upgraded UF CIS deposition system to enable fabrication of CIGS.

Table 1. Summary of PV performance on different metal substrates

\begin{tabular}{|c|c|c|c|c|c|c|c|}
\hline Substrate & $\begin{array}{c}\text { Voc } \\
(\mathrm{V})\end{array}$ & $\begin{array}{c}\text { Jsc } \\
\left(\mathrm{mA} / \mathrm{cm}^{2}\right)\end{array}$ & $\begin{array}{c}\text { FF } \\
(\%)\end{array}$ & $\begin{array}{c}\eta \\
(\%)\end{array}$ & $\begin{array}{c}\text { Rs } \\
(\Omega \mathrm{cm})\end{array}$ & $\begin{array}{c}\text { Rsh } \\
(\mathrm{k} \Omega \mathrm{cm})\end{array}$ & \\
\hline $\mathrm{Ni}$ & 0.465 & 17.243 & 37.936 & 3.037 & 16.684 & 0.097 & GD790-3 \\
\hline SS 302 & 0.643 & 16.392 & 52.753 & 5.555 & 13.900 & 0.358 & GD790-1 \\
\hline SS 304 & 0.643 & 16.392 & 52.753 & 5.555 & 13.900 & 0.358 & GD790-2 \\
\hline SS UT & 0.651 & 21.752 & 56.709 & 8.030 & 7.428 & 0.329 & GD790-4 \\
\hline
\end{tabular}

From best $3 \mathrm{Pm}$

\begin{tabular}{|c|c|c|c|c|c|c|c|}
\hline Substrate & $\begin{array}{c}\text { Voc } \\
(\mathrm{V})\end{array}$ & $\begin{array}{c}\text { Jsc } \\
\left(\mathrm{mA} / \mathrm{cm}^{2}\right)\end{array}$ & $\begin{array}{c}\text { FF } \\
(\%)\end{array}$ & $\begin{array}{c}\eta \\
(\%)\end{array}$ & $\begin{array}{c}\text { Rs } \\
(\Omega \mathrm{cm})\end{array}$ & $\begin{array}{c}\text { Rsh } \\
(\mathrm{k} \Omega \mathrm{cm})\end{array}$ & \\
\hline $\mathrm{Ni}$ & 0.537 & 9.994 & 52.184 & 2.801 & 34.575 & 0.154 & GD778-3 \\
\hline SS 302 & 0.610 & 15.883 & 51.341 & 4.976 & 11.457 & 0.267 & GD778-1 \\
\hline SS 304 & 0.665 & 15.962 & 55.207 & 5.860 & 13.269 & 0.347 & GD778-2 \\
\hline SS UT & 0.656 & 16.665 & 57.535 & 6.286 & 9.843 & 0.333 & GD778-4 \\
\hline
\end{tabular}

From best FF

\begin{tabular}{|c|l|l|l|l|l|l|}
\hline Substrate & $\begin{array}{l}\text { Good dots } \\
(\mathrm{FF}>45)\end{array}$ & $\begin{array}{l}\text { Shorted Dots } \\
(\mathrm{FF}<40)\end{array}$ & $\begin{array}{l}\text { Middle Dots } \\
(40<\mathrm{FF}<45)\end{array}$ & Total & Yield & \\
\hline SS 302 & 44 & 6 & 7 & 57 & $77 \%$ & GD790-1 \\
\hline SS 304 & 61 & 2 & 1 & 64 & $95 \%$ & GD790-2 \\
\hline Ni & 0 & 0 & 63 & 63 & $0 \%$ & GD790-3 \\
\hline SS UT & 55 & 8 & 1 & 64 & $85 \%$ & GD790-4 \\
\hline
\end{tabular}

\begin{tabular}{|c|l|l|l|l|l|l|}
\hline Substrate & $\begin{array}{l}\text { Good dots } \\
(\mathrm{FF}>45)\end{array}$ & $\begin{array}{l}\text { Shorted Dots } \\
(\mathrm{FF}<40)\end{array}$ & $\begin{array}{l}\text { Middle Dots } \\
(40<\mathrm{FF}<45)\end{array}$ & Total & Yield & \\
\hline SS 302 & 50 & 7 & 6 & 63 & $79 \%$ & GD778-1 \\
\hline SS 304 & 49 & 8 & 7 & 64 & $77 \%$ & GD778-2 \\
\hline Ni & 1 & 49 & 13 & 63 & $1.57 \%$ & GD778-3 \\
\hline SS UT & 64 & 0 & 0 & 64 & $100 \%$ & GD778-4 \\
\hline
\end{tabular}

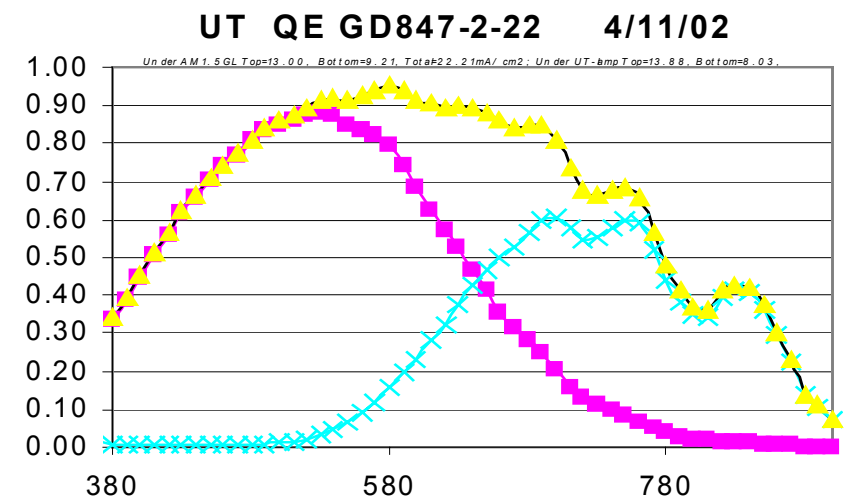

Figure 10. Quantum efficiencies of bottom \& top cells in fabricated tandem a-Si:Ge devices. 
While maintaining close ties with the Institute for Energy Conversion (IEC) at the University of Delaware, who have recently reported progress in several key areas of CIGS research (including deposition of high-performance cells onto lightweight polyamide substrates [Hanket et al. 2002]), we've also established a critical industry partnership with Daystar Technologies, whose founder (John Tuttle) has reported record efficiencies approaching 19\% in thin-film CIGS devices. Preliminary contact has also been established with Global Solar Industries, who has developed laser-scribing technology for fabricating integrated PV modules comprised of side-byside series-connected cells, similar to the proposed CIGS photoelectrode structure.

This year (Phase II) with the help of our industry and university partnerships, we have successfully completed the upgrade of our old CIS physical vapor deposition system to accommodate a gallium source. The upgraded system, pictured in Figures $11 \mathrm{a}$ and $11 \mathrm{~b}$, is capable of producing CIGS films over approximately a 4" x 4" area. A significant achievement has been the fabrication of CIGS photovoltaic devices onto flexible and light metal substrates (completed device shown in Figure 11c) with efficiencies exceeding 10\%. Near term goals include demonstration of near $14 \%$ on similar substrates. This year also, the modeling and validation experiments performed last year with physically-interconnected CIS devices were successfully repeated using CIGS devices.

(a)

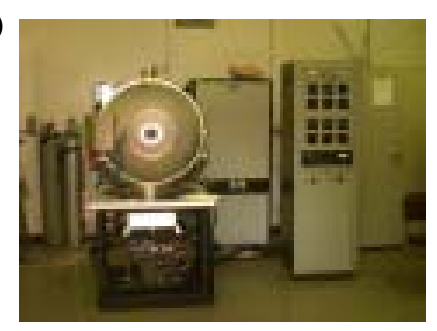

(b)

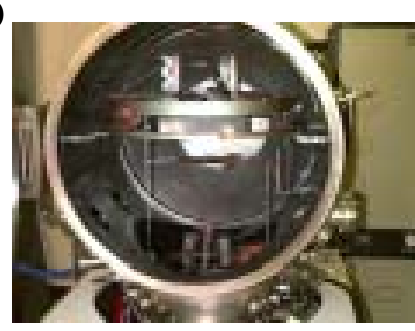

(c)

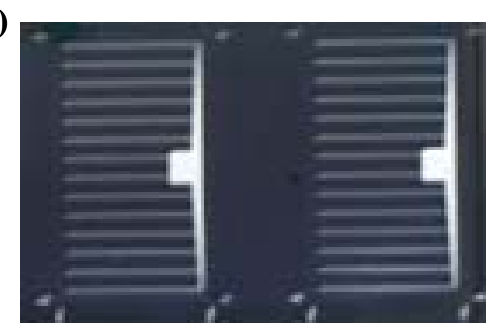

Figure 11. (a) CIGS deposition system at UH; (b) interior view ; (c) completed CIGS PV device.

\section{Hybrid Photoelectrode Development}

The main thrust of our Phase II research has been the development of the hybrid photoelectrode designs described in detail in the Introduction section. Significant milestones achieved to date in our Phase II hybrid development work can be summarized as follows:

- Established partnerships for development of $\mathrm{Fe}_{2} \mathrm{O}_{3}, \mathrm{WO}_{3}$ and $\mathrm{TiO}_{2}$.

- Performed initial modeling of hybrid photoelectrodes using published data for nanostructured $\mathrm{Fe}_{2} \mathrm{O}_{3}, \mathrm{WO}_{3}$ and $\mathrm{TiO}_{2}$ films.

- Selected $\mathrm{Fe}_{2} \mathrm{O}_{3}$ for initial hybrid photoelectrodes based on model results.

- Initiated program to develop $\mathrm{Fe}_{2} \mathrm{O}_{3}$ in-house, and with Duquesne University.

- Initiated fabrication of first prototype $\mathrm{Fe}_{2} \mathrm{O}_{3}$ hybrid devices.

The most important new partnership we established this year is with Dr. Shahed Khan at Duquesne University, who has demonstrated nanostructured $\mathrm{Fe}_{2} \mathrm{O}_{3}$ films with good photoactivity deposited by spray pyrolysis, and who is enthusiastic to participate in our hybrid photoelectrode research. Other important contacts have been made with Dr. Clara Santato at the University of 
Geneva, who has developed photoactive nanostructured $\mathrm{WO}_{3}$ films, and with Dr. Janus Nowotny at the University of New South Wales, who is interested in engineered forms of $\mathrm{TiO}_{2}$ for photoelectrochemical applications.

As indicated in the Introduction, presently available forms of dye-sensitized $\mathrm{TiO}_{2}$, which have near-ideal optical properties for hybrid photoelectrode use, are unstable under the OER. For near-term development, an alternative material is needed. Current literature concerning $\mathrm{WO}_{3}$ [Santato et al. 2001] and $\mathrm{Fe}_{2} \mathrm{O}_{3}$ [Khan \& Akikusa, 1999] indicate that forms of these wide-bandgap photoactive materials do remain stable for the OER. To evaluate their potential use, the previously described load-line analysis developed at UH needed modification. The new technique, which requires both the light $\mathrm{JV}$ and quantum efficiency characteristics of the photoactive interface, is illustrated in Figure 12. The design operating current of the device is dictated by efficiency goals, as per Equation 1. Referring to the curves in Figure 12, the light JV response of the PEC junction with respect to the normal hydrogen electrode (NHE) must be corrected to account for the current-dependent hydrogen overpotential loss occurring at the back surface of the device. The intersection of this corrected curve with the design current gives the required bias to be supplied by the underlying solid-state multijunction. This solidstate device must be designed to generate the design current at the required bias using the photons not absorbed in the PEC junction. This is where knowledge of the PEC quantum efficiency becomes critical.

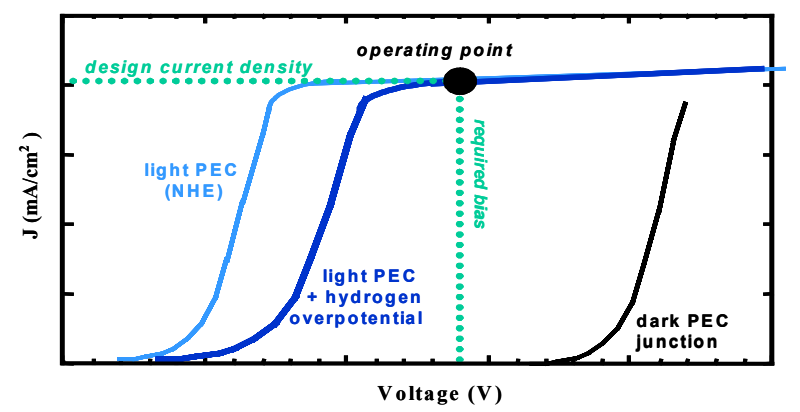

Figure 12. Load-line analysis of the hybrid photoelectrode configuration.

The model can be used to evaluate hybrid systems using, for example, nanostructured $\mathrm{WO}_{3}$ as the photoactive semiconductor. Figure 13 shows the photocurrent response $(13 a)$ and the quantum efficiency (13b) of $\mathrm{WO}_{3}$ nanostructured films prepared at the University of Geneva [Santato et al. 2001]. The HER overpotential correction to the light JV curve shown in Figure 13a is based on performance of the UH-developed thin film catalysts. In this JV plot, the peak photocurrent of this junction occurs at approximately $2.5 \mathrm{~mA} / \mathrm{cm}^{2}$. This low current can be understood in terms of the $\mathrm{WO}_{3}$ quantum efficiency curve in Figure 13b, which peaks around $85 \%$, but which spans only a narrow region in the blue wavelengths:. a spectrum consistent with the nominal bandgap of $2.7 \mathrm{eV}$ for tungsten trioxide. An important result from applying modeling techniques is that the operating point of $2.5 \mathrm{~mA} / \mathrm{cm}^{2}$ at a voltage bias of $1.5 \mathrm{~V}$ is readily achievable with a properly designed a-Si:Ge double-junction. 

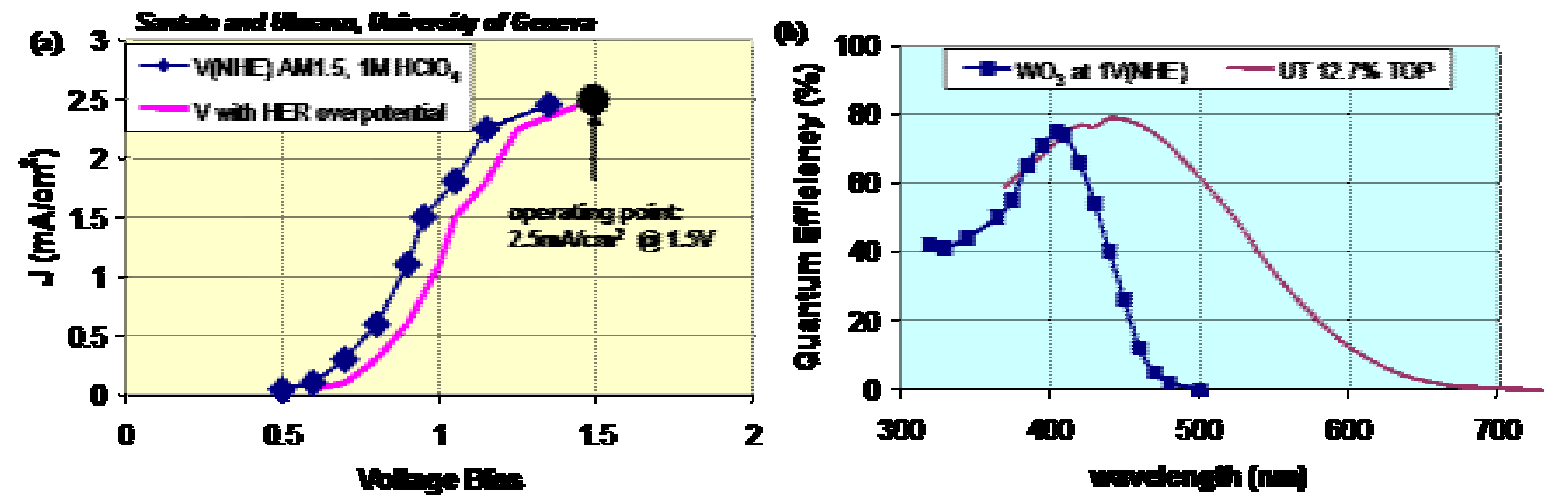

Figure 13. (a) Photocurrent response; (b) quantum efficiency of nanostructured $\mathrm{WO}_{3}$ films.

Also shown in Figure $13 \mathrm{~b}$ is the QE of the top cell of the Toledo $12.7 \%$ solid-state device. It is evident that replacing this top cell with a $\mathrm{WO}_{3}$ junction to form a hybrid photoelectrode does not extend the useful collection range of the device. As a result, the peak total photocurrent available remains at about $24 \mathrm{~mA} / \mathrm{cm}^{2}$, or $8 \mathrm{~mA} / \mathrm{cm}^{2}$ for each of the three junctions (two solidstate and one PEC) in the hybrid device. Unfortunately, the peak PEC photocurrent represents a bottleneck, limiting device current in the hybrid structure to $2.5 \mathrm{~mA} / \mathrm{cm}^{2}$, and STH efficiency to $3.1 \%$. Since the top PEC junction dictates performance, significant efficiency increases can be expected with material improvements in the $\mathrm{WO}_{3}$ photocurrent.

The performance of $\mathrm{Fe}_{2} \mathrm{O}_{3}$ in hybrid designs can also be evaluated using the model. AM 1.5 photocurrent response based on data published by Khan and Akikusa at Duquesne University [Khan \& Akikusa 1999] for their nanostructured iron oxide films is shown in Figure 14a, while quantum efficiency for these films is shown in Figure 14b. In contrast to the tungsten trioxide, current densities near the $8.1 \mathrm{~mA} / \mathrm{cm}^{2}$ goal are readily achievable in the nanostructured iron oxide, as seen in Figure 14a. Compared with $\mathrm{WO}_{3}, \mathrm{Fe}_{2} \mathrm{O}_{3}$ has a relatively low bandgap of 2.1 $\mathrm{eV}$, consistent with the observed spread in the quantum efficiency curve in Figure 14b. Although the QE never exceeds 23\%, integration of the AM 1.5 spectrum over a broad band of wavelengths accounts for the high levels of photogenerated current in the illuminated junction.
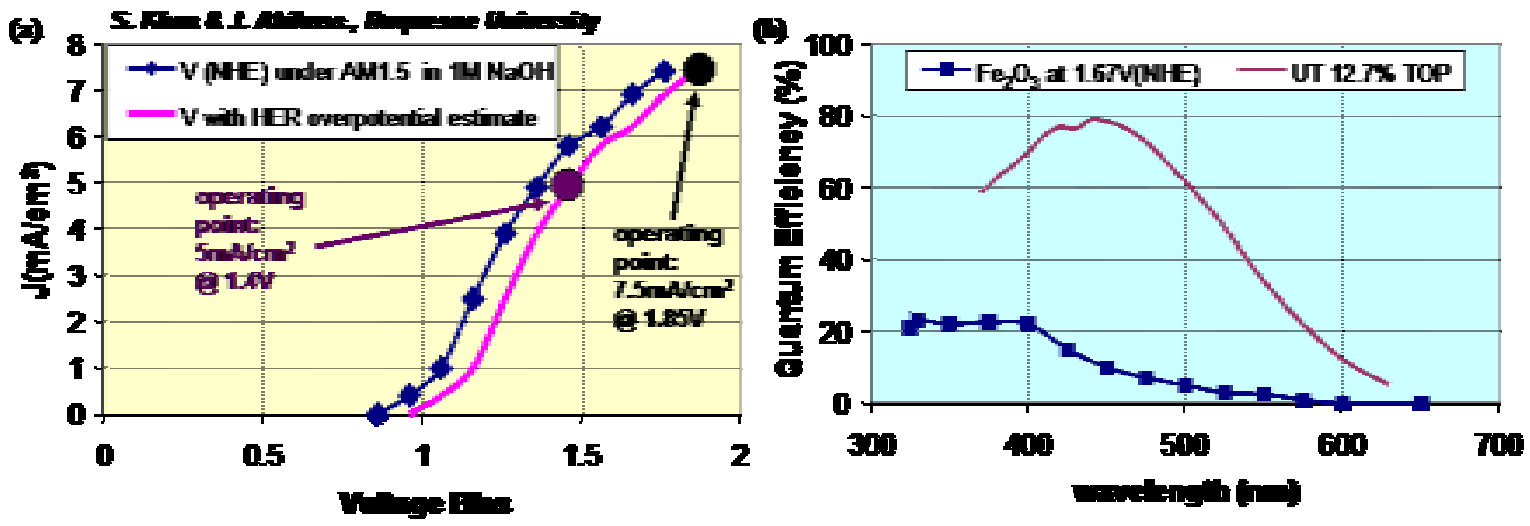

Figure 14. (a) Photocurrent response; (b) QE of nanostructured $\mathrm{Fe}_{2} \mathrm{O}_{3}$ films. 
A performance limitation in the iron oxide is not restricted current, but rather a high voltage bias constraint. For example, the operating point in Figure $14 \mathrm{a}$ of $7.5 \mathrm{~mA} / \mathrm{cm}^{2}$ at $1.8 \mathrm{~V}$ could not be driven by a double junction a-Si/a-Si:Ge or a-Si:Ge/a-Si:Ge device according to modeling results. A tandem solid-state cell at a reasonable bias of $1.5 \mathrm{~V}$ could drive the device at about 5 $\mathrm{mA} / \mathrm{cm}^{2}$ (about $6 \% \mathrm{STH}$ ), as indicated in the figure. It is evident, however, that nominal advances in iron oxide material properties could lead to significantly improved hybrid photoelectrode performance. With a reduction in required voltage bias at $7.5 \mathrm{~mA} / \mathrm{cm}^{2}$ from $1.8 \mathrm{~V}$ to 1.6 or $1.5 \mathrm{~V}$, a tandem, solid-state cell would be sufficient.

Based on it superior current characteristics over $\mathrm{WO}_{3}$ and its reported superior stability over dye-sensitized $\mathrm{TiO}_{2}$, as well as its material abundance and potential for low cost, we selected $\mathrm{Fe}_{2} \mathrm{O}_{3}$ as the material for initial photoelectrode development. Resulting from this decision, we've enlisted the support of $\mathrm{Dr}$. Khan at Duquesne, a pioneer in $\mathrm{Fe}_{2} \mathrm{O}_{3}$ development, who has agreed to deposit his photoactive material onto some of the prepared SS-UT/a-Si:Ge tandem/ITO substrates for hybrid photoelectrode testing. We have also initiated our own preliminary investigations into the behavior of reactively sputtered iron-oxide films. To date, we've observed good stability and high OER activity in sputtered $\mathrm{Fe}_{2} \mathrm{O}_{3}$ films, but no discernable photoactivity. Shown in Figure 15 are OER overpotentials of sputtered $\mathrm{Fe}_{2} \mathrm{O}_{3}$ deposited under different process conditions (15a), an SEM surface scan of a typical $\mathrm{Fe}_{2} \mathrm{O}_{3}$ sputtered at $\mathrm{UH}$ (15b), and an SEM surface scan of a photoactive $\mathrm{Fe}_{2} \mathrm{O}_{3}$ film deposited at Duquesne (15c).

(a)

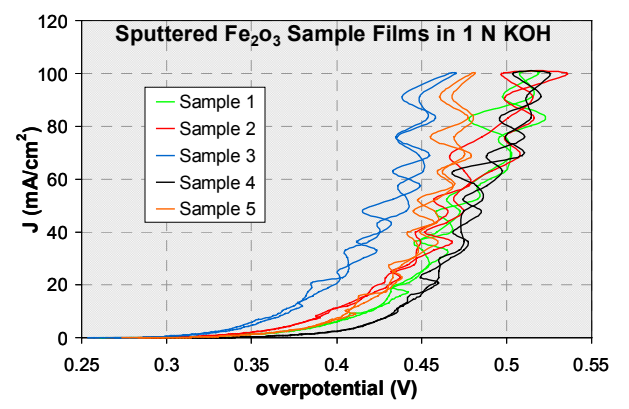

(b)

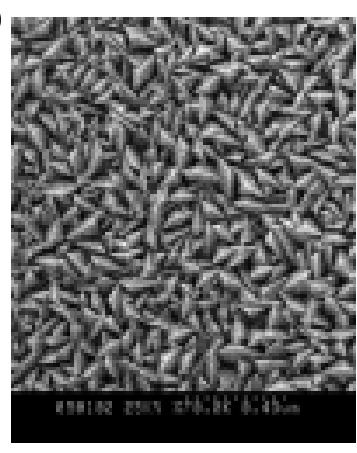

(c)

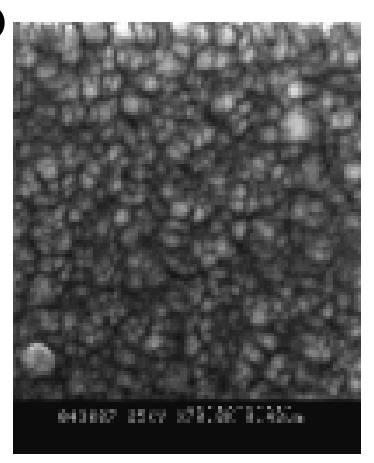

Figure 15. (a) OER overpotentials in sputtered $\mathrm{Fe}_{2} \mathrm{O}_{3}$; (b) SEM of sputtered $\mathrm{Fe}_{2} \mathrm{O}_{3}$; (c) SEM of photoactive spray-deposited $\mathrm{Fe}_{2} \mathrm{O}_{3}$.

Although there is a range in performance depending on process conditions, the OER activity for all the sputtered films shown in Figure $15 \mathrm{a}$ is generally high. Unfortunately, no bias reduction was observed in any of the sputtered samples under simulated AM1.5 light (ThermoOriel $1 \mathrm{~kW}$ simulator). There is, however, as seen in Figures $15 b$ and $15 c$ a marked difference in the surface morphologies of the non-photoactive sputtered films and the photoactive spraydeposited films from Duquesne. This could provide important clues in our quest for better understanding of iron-oxide photoelectrochemical interface, and for improved photoactive ironoxide films.

While waiting for Duquesne's processing of the prepared substrates for initial hybrid photoelectrode prototypes, we decided to process several prototypes at UH using the highest 
activity sputtered iron-oxide; although without a photoactive electrochemical interface, unbiased hydrogen photo-production was not expected in these devices. In order to evaluate photoelectrode stability and performance, the hydrogen and oxygen evolving surfaces were separated using the test jig shown schematically in Figure 16a, and pictured in Figure 16b. This test configuration allows insertion of instrumentation into the photoelectrode circuit to enable measurement of hydrogen current at different levels of applied bias.

As expected, unbiased hydrogen photogeneration was not observed in initial testing of the hybrid photoelectrodes fabricated with sputtered $\mathrm{Fe}_{2} \mathrm{O}_{3}$. However, hydrogen currents of approximately $3 \mathrm{~mA} / \mathrm{cm}^{2}$ were sustained under concentrated irradiance (about 2 suns) with a $1 \mathrm{~V}$ applied bias without observed degradation over the 1 hour duration of these tests. Although performance was poor in these un-optimized devices (non-photoactive electrochemical interface, and non-optimized optics), demonstration of stable operation in a $\mathrm{Fe}_{2} \mathrm{O}_{3}$ hybrid photoelectrode was a significant accomplishment.

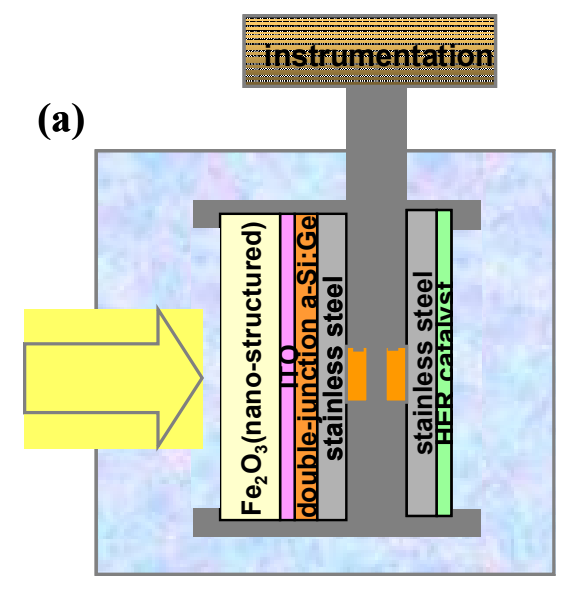

(b)

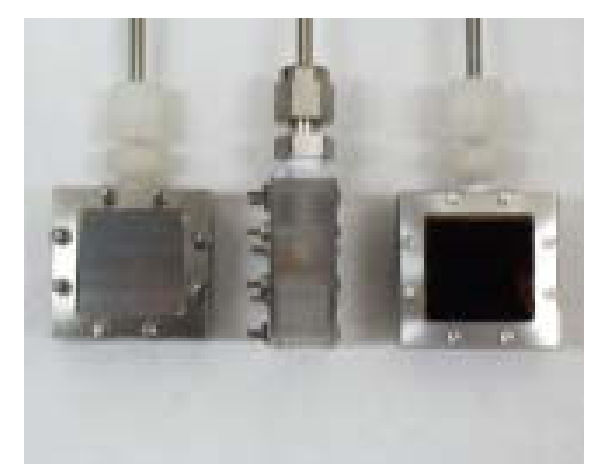

Figure 16. Photoelectrode testing jig for monitoring current under different biases: (a) cross section of design; (b) implementation with prototype hybrid photoelectrodes.

\section{CONCLUSIONS AND PLANS}

With this year's Phase II work, we have continued our emphasis on developing highperformance multi-junction hydrogen photoelectrodes based on low cost semiconductor and catalyst films. Significant progress was made in the advancement of amorphous silicon/germanium and copper indium gallium diselenide materials for solid-state photojunctions, and great strides were made in the research and development of the 'hybrid' photoelectrode concept, including important initial work on nanostructured wide-bandgap semiconductor films for the photoelectrochemical interface. As a result of preliminary research and modeling efforts, iron oxide was selected as the most promising material for incorporation into first prototype hybrid devices. We established a new partnership with Duquesne University, who has demonstrated capabilities in fabrication of nanostructured films of photoactive $\mathrm{Fe}_{2} \mathrm{O}_{3}$ deposited by the spray-pyrolysis method; At the same time, we initiated an in-house investigation into photoelectrochemical properties of reactively-sputtered iron oxide films. 
The Phase II work is progressing on-schedule; and initial sets of prototype hybrid devices based on tandem amorphous silicon/germanium coated with corrosion-resistant ITO and photoactive $\mathrm{Fe}_{2} \mathrm{O}_{3}$ are currently under fabrication. Near term plans include testing and analysis of this initial set of devices, followed by a redesign to better optimize interface and optical properties for a second stage prototype for fabrication and testing by the end of the contract period. Improved understanding of the $\mathrm{Fe}_{2} \mathrm{O}_{3}$ interface, which we plan to pursue in conjunction with Duquesne University, will be an important part of the optimization process. Re-design of the optical absorption characteristics in the a-Si:Ge solid state tandem devices for current matching with the photoelectrochemical interface (utilizing expertise of the University of Toledo) is also critical.

This year's work has also produced a new photoelectrode configuration combining the high efficiency of CIGS-based material design with the stability and simplicity of the hybrid concept. This new configuration, shown in Figure 17 is basically a hybrid triple junction photoelectrode using a high-efficiency CIGS-based tandem instead of the a-Si:Ge tandem. Development of CIGS tandem technology is still in early stages PV research, but with the recent upgrade of our CIGS deposition facilities and with our new industrial partnerships with CIGS experts such as Daystar, we will be well poised to take advantage of the anticipated technological advances to springboard into the next generation in hydrogen photoelectrodes.

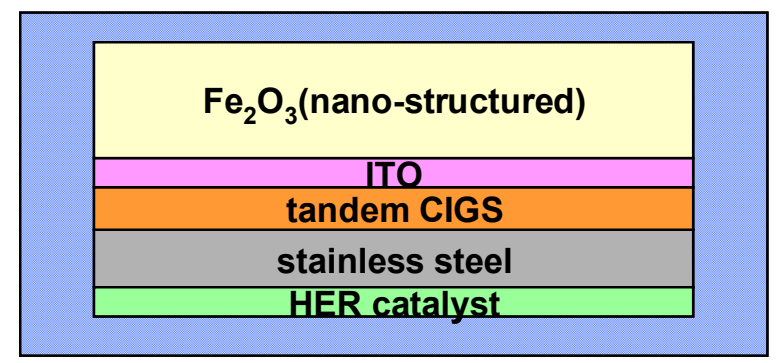

Figure 17. Next generation high-efficiency hybrid photoelectrode for hydrogen production based on monolithically-stacked CIGS-based tandem device.

\section{PUBLICATIONS LIST}

Conference presentations, published works, and patent disclosures generated to date during Phase II of this research project include:

- Conference Presentations

- International Conference on Advanced Materials (ICAM 2001): Invited Lecture on Hybrid Photoelectrode Design.

\section{- Journal Articles}

- "Design Considerations for a Hybrid Amorphous Silicon/Photoelectrochemical Multijunction Cell for Hydrogen Production" (accepted by the International Journal of Hydrogen Energy)

- "Evaluation of RF-Sputtered Indium-Tin Oxide Films for Photoelectrochemical Applications" (submitted to the Journal of the Electrochemical Society).

- Patent Disclosures

- "Hybrid Solid-State/Electrochemical Photoelectrode for Hydrogen Production". 


\section{ACKNOWLEDGMENTS}

We wish to thank the U.S. Department of Energy for support of this work under Grant DE-FG0494AL85804. We also thank Dr. Xunming Deng of the University of Toledo for fabrication of amorphous silicon samples, and Dr. Shahed Khan of the Duquesne University for fabrication of $\mathrm{Fe}_{2} \mathrm{O}_{3}$ samples.

\section{REFERENCES}

Chin, G. P. 2000. "Characterization and Optimization of Indium-Tin-Oxide (ITO) for Hydrogen Photoelectrodes." University of Hawaii Master's Thesis in Electrical Engineering.

Graetzel, M. 1999. “The Artificial Leaf, Bio-Mimetic Photocatalysis.” Catech, 3(1):4-16.

Hanket, G. et al., 2002. "Pilot-scale Manufacture of $\mathrm{Cu}(\operatorname{InGa}) \mathrm{Se} 2$ Films on a Flexible Polymer Substrate", In Proceedings of the $29^{\text {th }}$ IEEE Photovoltaics Specialists Conference, New Orleans, LA: IEEE.

Khan, S. U. M. and Akikusa, J. 1999 "Photoelectrochemical Splitting of Water at Nanocrystalline $\mathrm{n}-\mathrm{Fe}_{2} \mathrm{O}_{3}$ Thin-film Electrodes, Journal of Physical Chemistry B, 1999, 103: 7184-7189.

Konigstein, C. 1995. "Some Aspects of Photochemical Systems for Direct Light-induced Hydrogen Production”, Journal of Photochemistry and Photobiology A, 90: 141-152.

Miller, E. L., and R. E. Rocheleau. 1997. "Electrochemical Behavior of Reactively Sputtered Iron-doped Nickel Oxide." Journal of the Electrochemical Society, 144(9):3072-3077.

Miller, E. L., and R. E. Rocheleau. 1999. "Photoelectrochemical Hydrogen Production." In Proceedings of the 1999 U.S. Department of Energy Hydrogen Program Annual Review Meeting, Lakewood, CO: U.S. Department of Energy.

Miller, E. L., and R. E. Rocheleau. 2000. "Photoelectrochemical Hydrogen Production." In Proceedings of the 2000 U.S. Department of Energy Hydrogen Program Annual Review Meeting, San Ramon, CA: U.S. Department of Energy.

Miller, E. L., and R. E. Rocheleau. 2001. "Photoelectrochemical Hydrogen Production." In Proceedings of the 2001 U.S. Department of Energy Hydrogen Program Annual Review Meeting, Baltimore, MD: U.S. Department of Energy.

Rocheleau, R. E., and E. L. Miller. 1997. "Photoelectrochemical Production of Hydrogen: Engineering Loss Analysis." International Journal of Hydrogen Energy, 22(8):771-782.

Rocheleau, R. E., E. L. Miller, and A. Misra. 1998. "High-Efficiency Photoelectrochemical Hydrogen Production Using Multijunction Amorphous Silicon Photoelectrodes." Energy and Fuels 12:3-10.

Santato, C., Ulmann, M. and Augustynski, J. 2001, "Photoelectrochemical Properties of Nanostructured Tungsten Trioxide Films, Journal of Physical Chemistry B, 105: 936-940. 
Scaife, D. E., 1980. "Oxide Semiconductors in Photoelectrochemical Conversion of Solar Energy”, Solar Energy, 25: 41-54.

Swiss Federal Institute of Technology. 1996. "Dye-Sensitized Solar Cells Based on Nanocrystalline Oxide Semiconductor Films." <http://dcwww.efl.ch/icp/lCP-2/solarcellE.html>.

Tuttle, J., et al. 1995. "Thin-Film Cu(In,Ga)Se2 Materials and Devices: A Versatile Material for Flat Plate and Concentrator Photovoltaic Applications." Society of Photo-Optical Instrumentation Engineers, 2531:194-200.

Wang, W., X. B. Liao, S. Han, H. Povolny, X. B. Xiang, W. Du, and X. Deng. 2001. "TripleJunction a-Si-Based Solar Cells with All Absorber Layers Deposited at the Edge of a-Si to $\mu \mathrm{c}-\mathrm{Si}$ Transition." <http://www.physics.utoledo.edu/ dengx/ref3.pdf>. 